\title{
DETERMINACIÓN NO EXPERIMENTAL DE LA CONDUCTA SEDENTARIA EN ESCOLARES
}

\section{NOT EXPERIMENTAL DETERMINATION OF SEDENTARY BEHAVIOR IN SCHOOL-AGE CHILDREN}

\author{
Kristian Buhring B. (1), Patricio Oliva M. (2), Claudio Bravo C. (3) \\ (1) Departamento de Salud Pública, Facultad de Medicina, Universidad Católica de la Santísima Concepción. \\ (2) Facultad de Odontología, Universidad del Desarrollo. \\ (3) Escuela de Medicina, Universidad Católica de la Santísima Concepción. Chile.
}

\begin{abstract}
Sedentary lifestyle encourages the emergence of health problems among the population. The school population is at a stage of independence that stimulates the appearance of this condition. The definition of sedentary is unclear, as it is the classification of physical inactivity. Not only is defined as the absence of physical activity, but also considers leisure time activities. The purpose of this systematic review of literature is to describe the concepts related to the actual determination of sedentary life style in school. The results show that there is a definition of sedentary lifestyle associated with activities in school. However, there is no consensus on how to determine physical inactivity in school. There are no empirical methods for determining physical inactivity by recording the time spent on leisure activities that are fairly reliable.
\end{abstract}

Key words: systematic review; sedentary lifestyle; sedentary behavior; non-experimental measurement; school.

Este trabajo fue recibido el 9 de Septiembre de 2008 y aceptado para ser publicado el 10 de Enero de 2009.

\section{INTRODUCCIÓN}

Las investigaciones primarias en contadas ocasiones son capaces de dar una respuesta definitiva a cuestiones clínicas, obteniéndose resultados en la mayoría de las veces diferentes, incluso contradictorios. Sumado a lo anterior, el importante y sostenido incremento del número de publicaciones científicas, le han dado a la realización de revisiones de la literatura médica con lectura crítica (investigación secundaria), una importancia trascendental para la generación de evidencia tanto para la toma de decisiones en salud como para dar sustento a la creación de nuevos conocimientos.

Del sedentarismo como conducta no se ha llegado a una definición consensuada a nivel mundial, lo que lleva a dificultades intrínsecas para su diagnóstico y por lo tanto para su intervención, no obstante, existen algunas aproximaciones que son más aceptadas por la comunidad médica, en base a las cuales se han creado algunos sistemas de medición de esta conducta tanto de manera experimental como no experimental.
El impacto del sedentarismo en la población no deja de ser importante, considerando que es una de las condicionantes que contribuye a que por medio del bajo gasto energético de un individuo, en conjunto con ingestas alimentarias normales o elevadas, se provoque un desbalance energético que se puede traducir en alteraciones del estado de salud, como la malnutrición por exceso. Todo esto recrudece el impacto en la comunidad, originado de la pandemia en que se ha convertido la obesidad.

En la población escolar la situación no es diferente, incluso el sedentarismo en este grupo etario pudiese tener mayor importancia, puesto que a esa edad no sólo ocurren cambios fisiológicos, sino que también psicológicos y sociales, que se van moldeando de acuerdo a la conducta del escolar, que en definitiva son los determinantes de los hábitos de vida que trascienden a la adultez.

La vida sedentaria es frecuente durante la adolescencia (1), se ha sugerido que los niveles de actividad 
física de manera global tienden a disminuir durante esta etapa de la vida (2) lo que hace al paciente más proclive llegar a la edad adulta con una conducta sedentaria (3).

Las formas de medir el sedentarismo se pueden clasificar en métodos experimentales, representados por sistemas que intentan objetivar el gasto energético de una forma directa o indirecta, y no experimentales como lo son las escalas y/o cuestionarios que buscan cuantificar los tiempos destinados a actividades de bajo gasto energético a nivel individual y colectivo.

La medición del comportamiento sedentario ha recibido mucho menos atención que la actividad física en sí (4), y poco se ha publicado en la literatura con respecto a la fiabilidad y la validez de los datos que se obtienen al determinar el comportamiento sedentario (5).

Para prevenir la obesidad es necesario realizar múltiples intervenciones centradas en la actividad física, la dieta, el sedentarismo y el comportamiento entre los jóvenes $(6,7)$.

La actividad física es uno de los más importantes contribuyentes a la pérdida a largo plazo del tejido adiposo (8-10) y el manejo de peso (11).

En un reciente meta-análisis, el comportamiento sedentario (es decir, ver televisión y videos, uso de computadora) ha demostrado estar significativamente asociado con el aumento de peso y un descenso del nivel de actividad física en individuos estudiados (12). De hecho, algunos estudios encontraron que el uso de computadora está inversamente relacionado con la actividad física $(13,14)$.

Además de la relación con la inactividad física, la televisión también ha sido implicada como un factor que predispone para el sobrepeso y la obesidad en los jóvenes (15-17).

Robinson sugiere tres posibles mecanismos de vinculación de la televisión y la obesidad entre los niños y los jóvenes. Es la hipótesis de que la televisión: desplaza la actividad física y reduce el gasto energético, aumenta la ingesta de energía alimentaria al comer o durante la visualización de los efectos de la publicidad de alimentos, y disminuye la tasa metabólica en reposo durante su visualización (18).

Por lo tanto, es importante identificar los factores relacionados con la falta de actividad física en la adolescencia para una óptima prevención del sedentarismo y la obesidad durante toda la vida.

A continuación se expone la estrategia de búsqueda utilizada para acceder a la literatura relevante sobre la determinación del nivel de sedentarismo en escolares de una manera no experimental.

\section{MATERIAL Y MÉTODOS \\ Pregunta de búsqueda}

Para la revisión sistemática se planteó la siguiente pregunta de búsqueda: ¿Cómo determinar el nivel de sedentarismo en escolares de una manera no experimental?

\section{Objetivo de búsqueda}

Determinar el nivel de sedentarismo en escolares de una manera no experimental.

\section{Aplicación de criterios de selección Criterios de inclusión}

Todos los diseños metodológicos. Publicaciones en inglés, español, portugués y alemán.

Estudios realizados en los últimos 10 años. Edades entre 6 y 18 años. Ambos sexos. Determinación del sedentarismo de forma no experimental.

\section{Criterios de exclusión}

Estudios que no respondan a los idiomas indicados. Determinación del sedentarismo de forma experimental.

\section{Estrategia de búsqueda para la identificación de los estudios}

Se realizó búsquedas en Pubmed y BVS (Biblioteca Virtual en Salud), en las bases de datos EBSCO, Cochrane Library, ScienceDirect, SciELO y LILACS, revisiones narrativas y revisión de la literatura gris. Se efectuó búsquedas en las bases de datos electrónicas, combinada con las siguientes palabras claves: "sedentary", "sedentarism","physical inactivity", "behavioral", "measurement", "student", "questionnaire", "scales". La búsqueda en Pubmed se realizó con los siguientes límites: humans, male, female, english, spanish, child: 6-12 years, adolescent: 13-18 years. Cabe señalar que no se encontró "sedentarismo" como término Mesh. Se utilizó los términos booleanos "AND" y "OR" en la búsqueda. Todos los artículos permitieron ampliar la búsqueda por medio del Link "related articles". La búsqueda en BVS consideró bases de datos de Ciencias de la Salud en general: LILACS, MEDLINE, La Biblioteca Cochrane, SciELO; áreas especializadas: ADOLEC, BBO, BDENF, DESASTRES, HISA, HOMEOIDEX, LEYES, MEDCARIB, REPIDISCA; otros organismos internacionales: PAO, WOLIS. La búsqueda en EBSCO se desarrolló seleccionando las bases de datos Academic Search Premier, CINAHL con Full Text, Fuente Académica, MEDLINE con Full Text, SPORTDiscus con Full Text. Todas las búsquedas siguieron la misma estrategia descrita anteriormente. El proceso de revisión 
bibliográfica y análisis narrativo de los textos se centró en bibliotecas de las siguientes organizaciones: OMS, OPS y MINSAL (Ministerio de Salud de Chile). Este procedimiento fue realizado independientemente por dos investigadores con formación en epidemiología, revisiones sistemáticas y lectura crítica, dirimiéndose las diferencias por consenso.

\section{Análisis crítico de la literatura}

Se realizó análisis crítico utilizando las guías CASPe (Critical Appraisal Skills Programme en Español), para los diseños que cuentan con guía para su análisis.

\section{RESULTADOS \\ Definición de sedentarismo y beneficios de la actividad física}

Las definiciones de sedentarismo planteadas son variadas, algunas investigaciones sugieren que cumplen con esta característica aquellos individuos que practican actividad física con una frecuencia menor a tres veces por semana, con una duración menor a veinte minutos por cada una de esas sesiones $(19,20)$. Otra más actual, lo define cuando se realiza durante un tiempo menor a trescientos minutos por semana en momentos de ocio o en actividad cotidiana $(21,22)$.

Otra definición propuesta es una forma más precisa y aceptada que corresponde a estimar la totalidad del gasto energético diario en función del peso corporal, y derivar el diagnóstico de sedentarismo como el porcentaje del consumo energético realizado en actividades que requieren al menos cuatro equivalentes metabólicos (MET); que es equivalente de manera aproximada a la energía utilizada al caminar a paso rápido; del consumo total, se considera con esta condición cuando se utiliza menos del $10 \%$ de éste (23).

Una variante de esta última es la que considera las actividades con el mismo gasto energético (4 MET) en relación porcentual con el total, pero en tiempos de ocio (24).

Estas últimas son determinadas de una manera experimental, utilizando para esto instrumentos de medición directa o indirecta del gasto energético en un tiempo transcurrido de realización de algún ejercicio o actividad.

Finalmente, otra de las definiciones que recomienda la OMS, considera a las personas con el estilo de vida en cuestión cuando se invierte diariamente menos de veinticinco y treinta minutos en mujeres y hombres, respectivamente, en actividades de ocio que consuman cuatro o más MET $(20,25)$.

Cabe destacar que un MET es el consumo de energía de un individuo en reposo (aproximadamente 1Kcal/
$\mathrm{Kg}$ /hora) (26).

La utilización del concepto sedentarismo en la edad escolar, deriva de estudios realizados en población adulta y adolescente, puesto que no ha sido suficientemente investigado como fenómeno conductual en aquel grupo etario.

El incremento del sedentarismo comienza la mayoría de las veces en la adolescencia y continúa aumentando en la medida que avanza en edad la persona (25).

Múltiples investigaciones a nivel mundial han demostrado que la prevalencia del sedentarismo en todas las edades, incluida la escolar, ha ido en incremento sostenido independiente de la situación económica del país evaluado, por lo que se ha considerado un problema emergente y prioritario en salud, sobre todo teniendo en cuenta los efectos que esta conducta tiene, en el caso de la población pediátrica, tanto de manera inmediata como en la adultez.

Chile no es excepción de este cambio epidemiológico respecto del estilo de vida sedentario, entre las razones de este fenómeno se esgrimen la presencia de elementos de entretención sucedáneos de la actividad física, como computadoras, televisión, videojuegos, cambios en el hábito alimenticio y la mejora en la situación económica del país $(27,28)$.

La importancia de la realización de actividad física, en la población general, radica en los efectos beneficiosos sobre múltiples aspectos de las personas, por ejemplo, mejora la capacidad cardiovascular para la práctica de ejercicios que subsecuentemente lleva a la reducción del riesgo coronario, disminución de la fatiga y mejora la capacidad de trabajo físico; regula la composición corporal, lo que redunda en el decremento del riesgo de padecer obesidad, hipertensión arterial, enfermedad coronaria y diabetes mellitus tipo 2 ; además incrementa la masa muscular y flexibilidad corporal, relacionadas con la reducción de la presencia de enfermedades traumáticas, discapacidades (reduce el riesgo de lumbago crónico), osteoporosis y fracturas; disminuye el riesgo de cáncer de mama, próstata, colon y riñón; y además ayuda a prevenir enfermedades psiquiátricas $(29,30)$. En consecuencia, cada uno de estos beneficios confluye en la mejora de la calidad de vida (31-34), la sobrevida, disminución de mortalidad por todas las causas y hospitalizaciones (29,35-37), y por supuesto, reduce los costos en salud $(38,39)$.

El instaurar el hábito de la actividad física en la infancia tiene un efecto positivo sobre los pacientes, no sólo durante la niñez, sino que también trasciende a edades posteriores, este es un hecho demostrado y afirmado por instituciones gubernamentales de diversos países y por instituciones como la OMS. La ausencia del 
estilo de vida sedentario sobre la población pediátrica, tiene efecto sobre la capacidad física, bienestar y ausencia de enfermedades, óptimo desarrollo y crecimiento, además promueve el surgimiento de un estilo de vida activo que se mantiene a lo largo de la vida. Dicho de otra forma, aquellas personas que en la infancia fueron activos, tienen mayor probabilidad de continuar libres del sedentarismo durante la adultez $(40,41)$.

Se ha demostrado que la actividad física en individuos jóvenes disminuye el surgimiento de enfermedades crónicas que generalmente son diagnosticadas en la adultez. El efecto positivo que tiene la actividad física sobre la salud mental es otro aspecto a considerar, pero aún no está suficientemente demostrado, entre estos efectos se incluyen el bienestar psicológico, reducción de síntomas depresivos o ansiosos, y elevación de la autoestima especialmente en pacientes discapacitados (42). Cuando la actividad física es estructurada, puede desarrollar habilidades sociales y principios morales $(22,41,43,44)$. La importancia de mantener de manera permanente el hábito deportivo, radica en que el efecto es actual y no futuro, es decir, si una persona fue deportista de alto rendimiento en la adolescencia y después dejó de serlo y se convirtió en sedentario, el riesgo de padecer las enfermedades asociadas a este estilo de vida es el mismo que el resto de la gente que nunca practicó deporte alguno (25).

El sedentarismo no es sólo la ausencia de actividad física, sino que se debe considerar la intensidad de esta. Se han definido como actividades sedentarias, las realizadas en tiempo de ocio, como lo son el ver televisión, jugar videojuegos, el uso de la computadora (45).

\section{Recomendaciones de actividad física}

La recomendación del programa de promoción en salud de Inglaterra, considera que todos los niños deben realizar actividad física al menos de moderada intensidad de treinta minutos a una hora por día, siendo esta última lo ideal. El Centro para el Control de Enfermedades (CDC) de Atlanta $(31,37)$ y el American Collage of Sport Medicine (ACSM) (37) recomiendan una actividad física moderada durante al menos treinta minutos diarios continuos o fraccionados cada diez minutos. La American Academy of Pediatrics (AAP) también recomienda que los niños en edad escolar realicen a lo menos sesenta minutos de actividad física por día y además precisa que debería limitar actividades sedentarias a no más que dos horas por día $(42,46)$.

\section{Medición del sedentarismo}

Existen varias vías para cuantificar el sedentarismo, está la manera experimental (la más objetiva pero la menos práctica), que se puede llevar a cabo mediante la medición de la energía ocupada gracias al registro de la frecuencia cardiaca, el consumo de oxigeno, podómetro, acelerómetro y uno de los método más preciso, la del agua doblemente marcada. La forma más aplicable en la práctica clínica diaria es la de medición con escalas o cuestionario, a saber, está el auto reporte de percepción de nivel de actividad, cuestionarios para cuantificar el nivel de actividad física, reporte o medición de actividades realizadas durante tiempos de ocio, y cantidad de tiempo destinado a ejercicios extenuantes. No obstante, la medición del sedentarismo, continúa siendo una odisea debida básicamente a dos problemas, por un lado se carece de una definición uniforme del concepto, y por el otro, existen múltiples métodos propuestos para evaluarlo, siendo ninguno de ellos suficientemente práctico y a la vez preciso (19).

La cuantificación de manera práctica de este estilo de vida en la población pediátrica, es crucial para ir evaluando la situación epidemiológica del lugar geográfico de interés y también para pesquisar los individuos que padecen de este factor de riesgo potencialmente modificable e intervenirlo. Un cuestionario diseñado para individuos en edad adolescente, que tiene un formato para ser auto aplicado, se denomina Adolescent Sedentary Activities Questionnaire (ASAQ), el que evalúa cinco dimensiones que se detallan claramente, de las que derivan diez u once ítems, en los que se registra el tiempo ocupado en un número de actividades sedentarias previamente definidas (utilización de menos de 1,5 MET). Entre las actividades evaluadas están: ver televisión, ocupar videojuegos, DVD, computadora, juegos y comunicación por internet, tiempo de estudio, lectura, estar sentado con amigos, uso de teléfono, tocar instrumentos musicales, desplazamientos en vehículos motorizados, hobbies, actividades artísticas, y asistencia a iglesia o al colegio en fin de semana, todas estas actividades fuera de horario escolar. El ASAQ tiene una buena fiabilidad test re-test y hasta que mejores métodos para evaluar la validez de criterio sean desarrollados, parece ser el mejor instrumento disponible para evaluar el tiempo destinado a una gama de comportamientos sedentarios entre jóvenes fuera de horas de clase (47).

Un cuestionario diseñado para evaluar la actividad física en adolescentes durante la semana previa a la evaluación denominada Physical Activity Questionnaire (PAQ-C), tiene la limitación de no discriminar entre la intensidad, frecuencia y duración de las actividades físicas $(48,49)$.

Además que determina el sedentarismo de una forma indirecta ya que sólo se obtiene información del tiempo y la intensidad del ejercicio. Ya se ha descrito 
el método para obtener a través de una entrevista, las horas destinadas a actividades sedentarias la semana anterior a la aplicación de esta (50). La actividad sedentaria ha sido evaluada por preguntas en cuestionarios (51). Existen cuestionarios que determinan el sedentarismo validados en jóvenes estudiantes de secundaria $(52,53)$

\section{DISCUSIÓN}

Si bien existen definiciones objetivas del sedentarismo, no es posible obtener una que tienda a unificar el criterio diagnóstico de esta condición, situación que ocurre tanto en la población adulta como infantil. La utilización del concepto de sedentarismo en la edad escolar deriva de las investigaciones en adultos, por lo tanto hay que tener precaución al extrapolar estos datos a la población en edad escolar, puesto que no es metodológicamente adecuado hacer aquello.

El sedentarismo ha tenido un incremento sostenido a nivel mundial, indistintamente del género, edad y situación socioeconómica, lo que se traduce en un problema emergente tanto de manera local como global.

En la edad adulta se han arraigado fuertemente los hábitos adquiridos durante la infancia, y la edad escolar la última oportunidad en la que se puede intervenir para evitar la incorporación de este vicio conductual. Para esto, la identificación del sedentarismo como conducta, y no sólo la no realización de la actividad física definida como tal, puede ser determinante en la intervención para evitar esta conducta como determinante de la salud. El sedentarismo ocasiona problemas de salud y estos repercuten en los gastos de los sistemas sanitarios. Por otro lado las personas que son activas físicamente en etapas de la infancia, tienden a tener una mayor probabilidad de estar libres del sedentarismo en edades adultas. Existen varias formas de determinar el nivel de actividad física y sedentarismo, que se agrupan en experimentales y no experimentales. Ambas tienen sus ventajas y desventajas, pero está demostrado que la determinación no experimental es un mecanismo fácilmente aplicable, de bajo costo y con una confiabilidad en sus resultados que lo hacen factible de realizar para esta determinación. Esta cuantificación práctica de este estilo de vida en la población pediátrica, es determinante para evaluar la situación epidemiológica de este grupo etario y para pesquisar los individuos que padecen de sedentarismo e intervenirlos en el momento adecuado. El Adolescent Sedentary Activities Questionnaire (ASAQ), demuestra ser un instrumento optimo para este propósito, ya que aparte de ser de fácil aplicación demostró tener buena fiabilidad test re-test.

\section{CONCLUSIONES}

El sedentarismo es actualmente una condicionante de salud y en aumento en toda la población, independiente de factores sociales, económico, genérico, entre otros. Es un problema que ocasiona trastornos en la salud de los pacientes, así como incremento en los gastos en salud. La determinación del sedentarismo es una acción prioritaria para la salud pública, en las actividades de intervención, prevención y promoción en salud. La edad escolar es posiblemente la última instancia en la que las intervenciones logren revertir los niveles de sedentarismos en la población de una manera efectiva, lo que explica la importancia de la detección precoz de esta conducta.

La determinación no experimental viene a ser un procedimiento fácil de aplicar, económico, no invasivo y efectivo para detectar este problema.

\section{RESUMEN}

El sedentarismo favorece la aparición de problemas de salud en la población. La población escolar está en una etapa de independencia que genera la aparición de esta condición. La definición de sedentarismo está poco clara, lo mismo ocurre con la clasificación de sedentarismo. No solo se define como la ausencia de actividad física, también considera actividades destinada al tiempo de ocio. La manera en que se determina el sedentarismo, es identificando aquellos individuos que son inactivos. El propósito de esta revisión es describir los conceptos relacionados con la determinación no experimental del sedentarismo en escolares. Se realiza una revisión sistemática de la literatura. Los resultados muestran que existe una definición de sedentarismo y se la asocia a escolares. La forma de determinar el sedentarismo en escolares no está consensuada. Existen métodos no experimentales que permiten determinar el sedentarismo, registrando el tiempo destinado a actividades de ocio que son bastante confiables.

Palabras claves: revisión sistemática; sedentarismo; conducta sedentaria; medición no experimental; escolares.

Dirigir la correspondencia a:

Dr.

Kristian Buhring Bonacich

Departamento de Salud Pública

Facultad de Medicina

Universidad Católica de la Santísima Concepción

Alonso de Ribera 2850

Concepción

Teléfono: 56-41-2735416

Fax: 56-41-2735401

E mail: kbuhring@ucsc.cl 


\section{BIBLIOGRAFÍA}

1. Andersen, R.E. Crespo, C.J. Bartlett, S.J. Cheskin, L.J. Pratt, M. Relationship of physical activity and television watching with body weight and level of fatness among children: results from the third national health and nutrition examination survey, JAMA 1998; 279 (12), 938-942.

2. Trost S.G. Pate R.R. Sallis J.F, et al. Age and gender differences in objectively measured physical activity in youth. Med Sci Sports Exerc 2002; 34:350-5.

3. Tammelin T, Nayha S, Laitinen J, et al. Physical activity and social status in adolescence as predictors of physical inactivity in adulthood. Prev Med 2003; 37:375-81.

4. Dietz WH. The role of lifestyle in health: The epidemiology and consequences of inactivity, Proc Nutr Soc 1996; 55:829-40.

5. Penny G, Melissa C. Nelson, RD, Barry M. Popkin. Longitudinal Physical Activity and Sedentary Behavior Trends Adolescence to Adulthood. Am J Prev Med 2004; 27(4)277-283.

6. Dietz, W.H., Gortmaker, S.L. Preventing obesity in children and adolescents. Annu Rev Public Health 2001; 22, 337-353.

7. Baranowski, T., Cullen, K.W., Nicklas, T., Thompson, D., Baranowski, J. School-based obesity prevention: a blueprint for taming the epidemic. Am J Health Behav. 2002; 26 (6), 486-493.

8. Pronk, N.P., Wing, R.R. Physical activity and longterm maintenance of weight loss. Obes Res 1994; 2, 587-599.

9. Jakicic, J.M., Marcus, B.H., Gallager, K.I., Napolitano, M., Lang,W.,. Effect of exercise duration and intensity on weight loss in overweight, sedentary women; a randomized trial. J Am Med Assoc 2003; 290(10), 1323-1330.

10. Schoeller, D.A., Shay, K., Kushner, R.F. How much physical activity is needed to minimize weight gain in previously obese women? Am J Clin Nutr 1997; 66, 551-556.

11. Steinbeck, K.S. The importance of physical activity in the prevention of overweight and obesity in childhood: a review and an opinion. Obes Rev 2001; 2 , 117-130.

12. Marshall SJ, Biddle SJ, Gorely T, et al. Relationships between media use, body fatness and physical activity in children and youth: a meta-analysis. Int J Obes Relat Metab Disord 2004; 28:1238-46.

13. Strauss RS, Rodzilsky D, Burack G, Colin M. Psychosocial correlates of physical activity in healthy children. Arch Pediatr Adolesc Med 2001; 155:897902.
14. Fotheringham MJ, Wonnacott RL, Owen N. Computer use and physical inactivity in young adults: public health perils and potentials of new information technologies. Ann Behav Med 2000; 22:26975.

15. Eisenmann JC, Bartee RT, Wang MQ. Physical activity, TV viewing, and weight in U.S. youth: 1999 Youth Risk Behavior Survey. Obes Res 2002; 10:379-85.

16. Crespo CJ, Smit E, Troiano RP, et al. Television watching, energy intake, and obesity in US children: results from the third National Health and Nutrition Examination Survey, 1988-1994. Arch Pediatr Adolesc Med 2001; 155:360-5.

17. Lowry R, Wechsler H, Galuska DA, et al. Television viewing and its associations with overweight, sedentary lifestyle, and insufficient consumption of fruits and vegetables among US high school students: differences by race, ethnicity, and gender. J Sch Health 2002; 72:413-21.

18. Robinson TN. Television viewing and childhood obesity. Pediatr Clin North Am 2001; 48:1017-25.

19. Ricciardi R. Sedentarism: a concept analysis. Nursing Forum. 2005; 40:79-87.

20. Cabrera A, Rodríguez M, Rodriguez L, Anía B, Brito B, Muros M, Almeida D, Batista M, Aguirre A. Sedentary lifestyle: physical activity duration versus percentage of energy expenditure. Rev Esp Cardiol. 2007; 60(3):231-3.

21. Biddle S, Cavill N, Sallis J. Young and active? Young people and health-enhancing physical activity-evidence and implications. London: Health Education Authority, 1998.

22. Curi P, Dâmaso A, Gonçalves H, Gomes C. Prevalência de sedentarismo e fatores associados em adolescentes de 10-12 anos de idade. Cad Saúde Pública 2006; 22(6):1277-1287.

23. Bernstein SM, Morabia A, Sloutskis D. Definition and prevalence of sedentarism on an urban population. Am J Public Health 1999; 89:862-27.

24. Varo JJ, Martínez-González MA, De Irala-Estévez J, Kearney J, Gibney M, Martínez JA. Distribution and determinants of sedentary lifestyles in the European Union. Int J Epidemiol 2003; 32:138-46.

25. World Health Organization. Health and Development through Physical Activity and Sport, 2003.

26. Ainsworth BE, Haskell WL, Leon AS, Jacobs DR Jr, Montoye HJ, Sallis JF, et al. Compendium of physical activities: energy costs of human movement. Med Sci Sports Exerc 1993; 25:71-80.

27. Barria RM, Amigo H. Transición nutricional: una revisión del perfil latinoamericano. Arch Latinoam 
Nutr 2006; 56: 3-11.

28. Olivares S, Kain J, Lera L, Pizarro F, Vio F, Moron C. Nutritional status, food consumption and physical activity among Chilean school children: a descriptive study. Eur J Clin Nutr 2004; 58: 1278-85.

29. Kujala U, Kaprio J, Sarna S, Koskenvuo M. Relationship of Leisure-Time Physical Activity and Mortality the Finnish Twin Cohort. JAMA 1998; 279:440-444.

30. Slattery ML, Edwards SL, Ma KN, Friedman GD, Potter, JD. Physical activity and colon cancer: a public health perspective. Ann Epidemiol 1997; 7:137-45.

31. U.S. Department of Health and Human Service. Physical Activity and Health: A Report of the Surgeon General. Atlanta, GA: U.S. Department of Health and Human Services, Centers for Disease Control and Prevention, National Center for Chronic Disease Prevention and Health Promotion, 1996.

32. Penninx BW, Leveille S, Ferrucci L, van Eijk JT, Guralnik JM. Exploring the effect of depression on physical disability: longitudinal evidence from the established populations for epidemiologic studies of the elderly. Am J Public Health 1999; 89:134652.

33. Ferrucci L, Izmirlian G, Leveille $\mathrm{S}$ et al. Smoking, physical activity, and active life expectancy. Am J Epidemiol 1999; 149:645-53.

34. Province MA, Hadley EC, Hornbrook MC et al. The effects of exercise on falls in elderly patients. A preplanned meta-analysis of the FICSIT Trials. Frailty and Injuries: Co-operative Studies of Intervention Techniques. JAMA 1995; 273:1341-47.

35. Haapanen N, Miilunpalo S, Vuori I, Oja P, Pasanen M. Characteristics of leisure time physical activity associated with decreased risk of premature all-cause and cardiovascular disease mortality in middleaged men. Am J Epidemiol 1996; 143:87080.

36. Wei M, Kampert JB, Barlow CE, et al. Relationship between low cardiorespiratory fitness and mortality in normal-weight, overweight and obese men. JAMA 1999; 282:1547-53.

37. Pate RR, Pratt M, Blair SN, et al. Physical activity and public health. A recommendation from the Centers for Disease Control and Prevention and the American College of Sports Medicine. JAMA 1995; 273:402-07.

38. Haskell W, Montoye H, Orenstein D. Physical activity and exercise to achieve health-related physical fitness components. Public Health Rep 1985;
100:202-212.

39. Blair SN, Kohl HW, Paffenbarger RS, Clark DG, Cooper KH, Gibbons LW. Physical fitness and allcause mortality: A prospective study of healthy men and women. JAMA 1989; 262:2395-2401.

40. Parsons TJ, Power C, Logan S, Summerbell CD. Childhood predictors of adult obesity: a systematic review. Int J Obes Relat Metab Disord 1999; 23 Suppl 8:S1-107.

41. Kraut A, Melamed S, Gofer D, Froom P. Effect of school age sports on leisure time physical activity in adults: The CORDIS Study. Med Sci Sports Exerc 2003; 35:2038-42.

42. Floriani V, Kennedy C. Promotion of physical activity in children. Curr Opin Pediatr 2008; 20:90-95.

43. Twisk JW. Physical activity guidelines for children and adolescents: a critical review. Sports Med 2001; 31:617-27.

44. Gordon-Larsen P, Nelson MC, Popkin BM. Longitudinal physical activity and sedentary behavior trends: adolescence to adulthood. Am J Prev Med 2004; 27:277-83.

45. Sugiyama T, Xie D, Graham-Maar R, Inoue K, Kobayashi Y, Stettler N. Dietary and Lifestyle Factors Associated with Blood Pressure among U.S. Adolescents . Journal of Adolescent Health 2006; 40:166-172.

46. Council on Sports Medicine and Fitness and Council on School Health: Active healthy living: prevention of childhood obesity through increased physical activity. Pediatrics 2006; 117:1834-1842.

47. Hardy L, Booth M, Okely A. The reliability of the Adolescent Sedentary Activity Questionnaire (ASAQ). Preventive Medicine 2007; 45:71-74.

48. Crocker, P. R, Bailey, D. A, Faulkner, R. A, Kowalski, K. C, McGrath, R. Measuring general levels of physical activity: Preliminary evidence for the Physical Activity Questionnaire for Older Children. Medicine and Science in Sports and Exercise 1997; 29:1344-1349.

49. Kowalski, K, Crocker, P, Faulkner, R. Validation of the physical activity questionnaire for older children. Pediatric Exercise Science 1997; 9:174-186.

50. Gordon-Larsen P, McMurray RG, Popkin BM. Adolescent physical activity and inactivity vary by ethnicity: the National Longitudinal Study of Adolescent Health. J Pediatr 1999; 135:301-6.

51. Sirard J, Pfeiffer K, Pate R. Motivational factors associated with sports program participation in middle school students. J Adolescent Health 2006; 38:696-703.

52. Pereira MA, Fitzgerald SJ, Gregg EW, et al. A 
collection of physical activity questionnaires for health-related research: The Modifiable Activity Questionnaire for Adolescents. Med Sci Sports Exerc 1997; 29, S79-82.

53. Aaron DJ, Kriska AM, Dearwater SR, Cauley JA, Mets KF, Laporte RE. Reproducibility and validity of an epidemiologic questionnaire to assess past year physical activity in adolescents. Am J Epidemiol 1995; 142, 191-201.

54. Sallis JF, Haskell WL, Wood PD, et al. Physical activity assessment methodology in the five-city project. Am I Epidemiol 1985; 121:91-106. 